\title{
Alopecia Scoring: The Quantitative Assessment of Hair Loss in Captive Macaques
}

\author{
Paul E. Honess, ${ }^{1}$ Jessica L. Gimpel, ${ }^{2}$ Sarah E. Wolfensohn ${ }^{1}$ and Georgia J. Mason ${ }^{3}$ \\ 1Oxford University Veterinary Services, Oxford, UK; ${ }^{2}$ Fauna Australis, Departamento de Ciencias Animales, \\ Facultad de Agronomía e Ingeniería Forestal, Pontificia Universidad Católica de Chile, Santiago, Chile; \\ ${ }^{3}$ Department of Animal Science, University of Guelph, Guelph, Ontario, Canada
}

\begin{abstract}
Summary - Many captive animals show forms of pelage loss that are absent in wild or free-living conspecifics, which result from grooming or plucking behaviours directed at themselves or at other individuals. For instance, dorsal hair loss in primates such as rhesus macaques (Macaca mulatta) in research facilities, results from excessive hair-pulling or over-grooming by cage-mates. This behaviour appears to be associated with stress, and is controllable to some extent with environmental enrichment. Quantifying alopecia in primates (as in many species) is therefore potentially useful for welfare assessment. A simple system for scoring alopecia was developed and its reliability was tested. Study 1 showed high interobserver reliability between two independent scorers in assessing the state of monkeys' coats from photographs. Study 2 showed that there were no significant differences between the scores derived from photographs and from direct observations. Thus, where hair loss due to hair pulling exists in captive primates, this scoring system provides an easy, rapid, and validated quantitative method, for use in assessing the success of attempts to reduce it via improved husbandry. In the future, such scoring systems might also prove useful for quantifying barbering in laboratory rodents.
\end{abstract}

Key words: alopecia, animal welfare, hair loss, over-grooming, primates, scoring system, stress.

Address for correspondence: P.E. Honess, Oxford University Veterinary Services, Parks Road, Oxford OX1 3PT, UK.

E-mail: paul.honess@vet.ox.ac.uk

\section{Introduction}

Captive animals often show patterns of pelage loss (alopecia) that are rare or absent in wild or freeliving conspecifics. Typical avian examples include feather damage and loss in caged parrots, often around the head and neck (1), and in farmed poultry, usually from the body and around the cloacal vent (2). Typical mammalian examples include areas of shortened or missing fur on the tails of farmed American mink (3), and patches of thin or absent hair on the heads and shoulders of laboratory rodents $(4,5)$ and the backs and limbs of laboratory primates (6; Figure 1). Although alopecia can be affected by changes in hormonal status (for example, prolactin and melatonin levels) and by nutrition, ageing, and reproductive condition and/or disease (7), in captive animals it often has a behavioural cause, resulting, for instance, from hair-pulling. Such behaviour may represent excessive self-grooming (as is the case for single-housed parrots [8]) or allo-grooming (for example, laboratory mouse "barbering" [4]), or alternatively, may be derived from redirected sucking (as may be the case for mink [3]) or foraging (there are links between feather-eating and "pica" - the eating of non-edible objects - in poultry [9]).
Whatever the precise aetiology, for a number of reasons, over-grooming and pelage loss in captive animals are often considered a sign of poor welfare. Firstly, it is often more severe or more prevalent during long-term treatments, which independent evidence links with poor welfare (for example, early weaning in mink [3]; isolation versus social-housing for parrots [8]; alcohol intoxication for long-tailed macaques, Macaca fascicularis [10]; and barren and unstimulating housing conditions for other primates, such as pig-tailed macaques, $M$. nemestrina, [11]). Secondly, it appears to be similar to the human hair-plucking condition, trichotillomania (see 5), an obsessive-compulsive disorder which, like hair-plucking in non-human primates (hereafter called primates), is sometimes linked with psychological stress $(12,13)$ and can often be alleviated by anti-depressive drugs (14-16). Both these conditions appear to be compulsive, and entail the plucking of one's own or another's hair which may then be orally manipulated (17), and then eaten by primates, despite being indigestible (13). Finally, it may cause, as well as reflect, poor welfare, via transient pain in plucked animals (4) or enhanced rates of heat loss due to reduced insulation (18). Furthermore, in poultry, the resulting skin damage, which is vulnerable to secondary infection, may lead to additional injurious pecking or even to 
Figure 1: Photographs illustrating the five-point scoring scale for alopecia in primates

a)

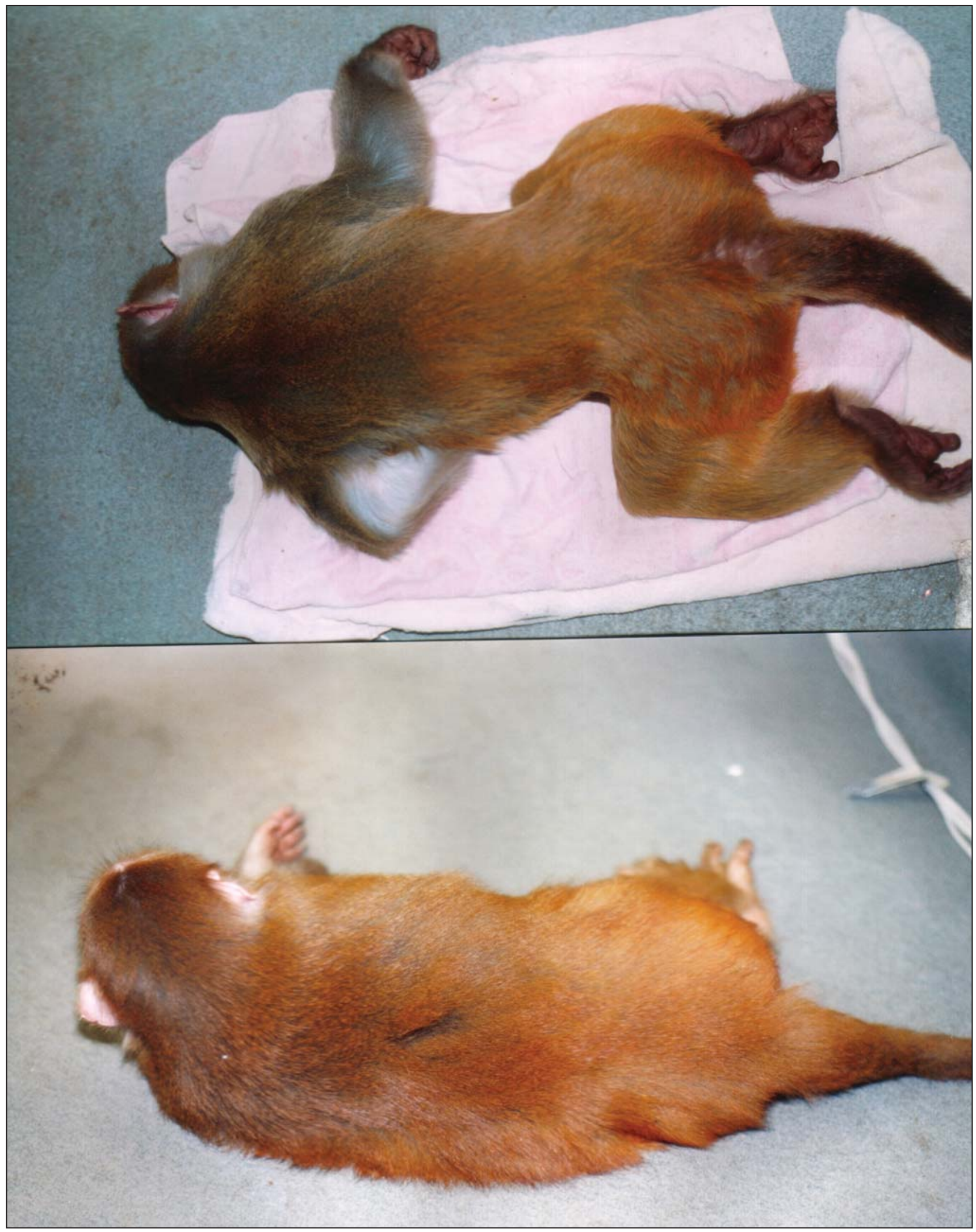

a) Score 1: very good coat condition 


\section{Figure 1: continued}

b)

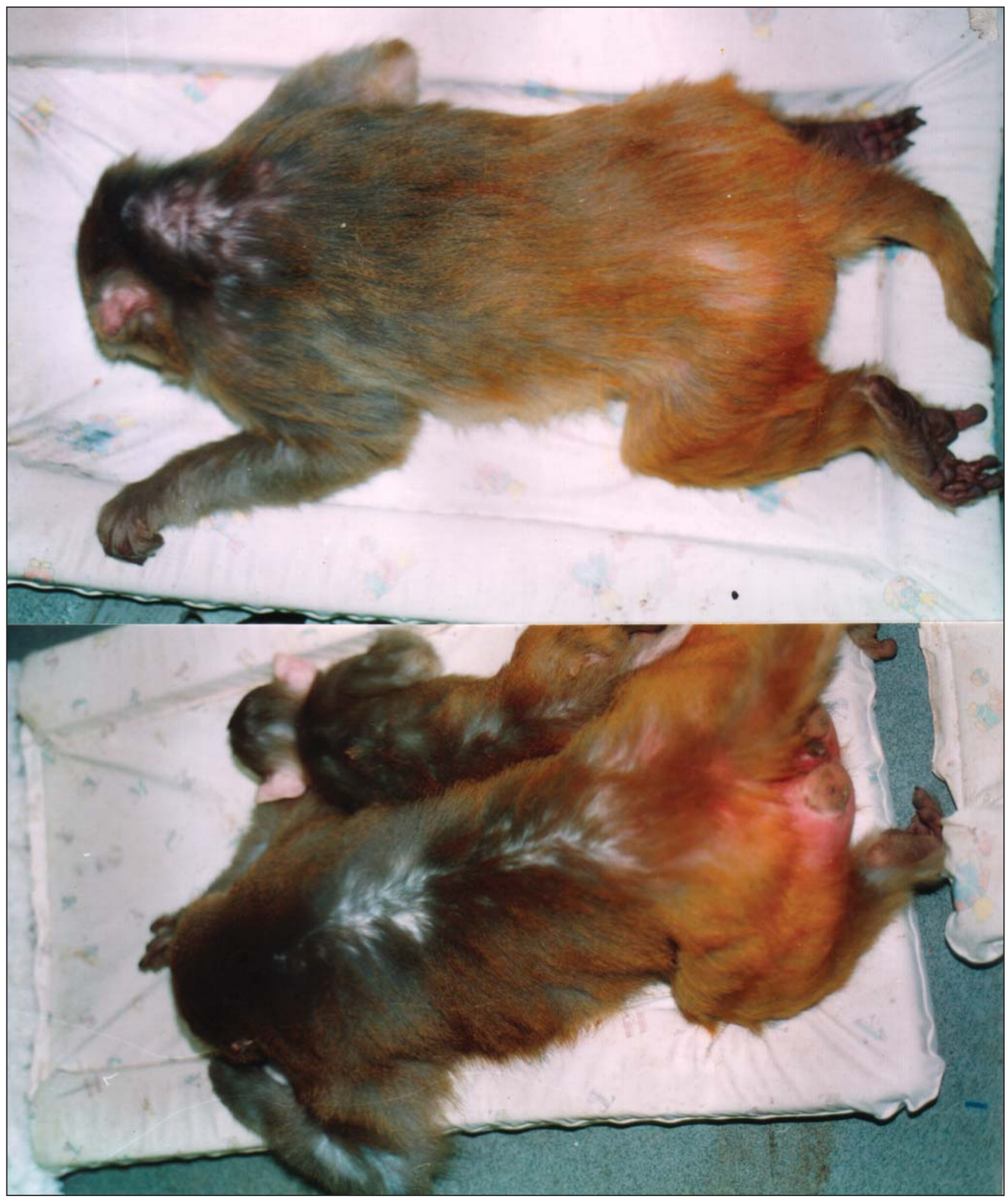

b) Score 2: few small patches of alopecia 


\section{Figure 1: continued}

c)

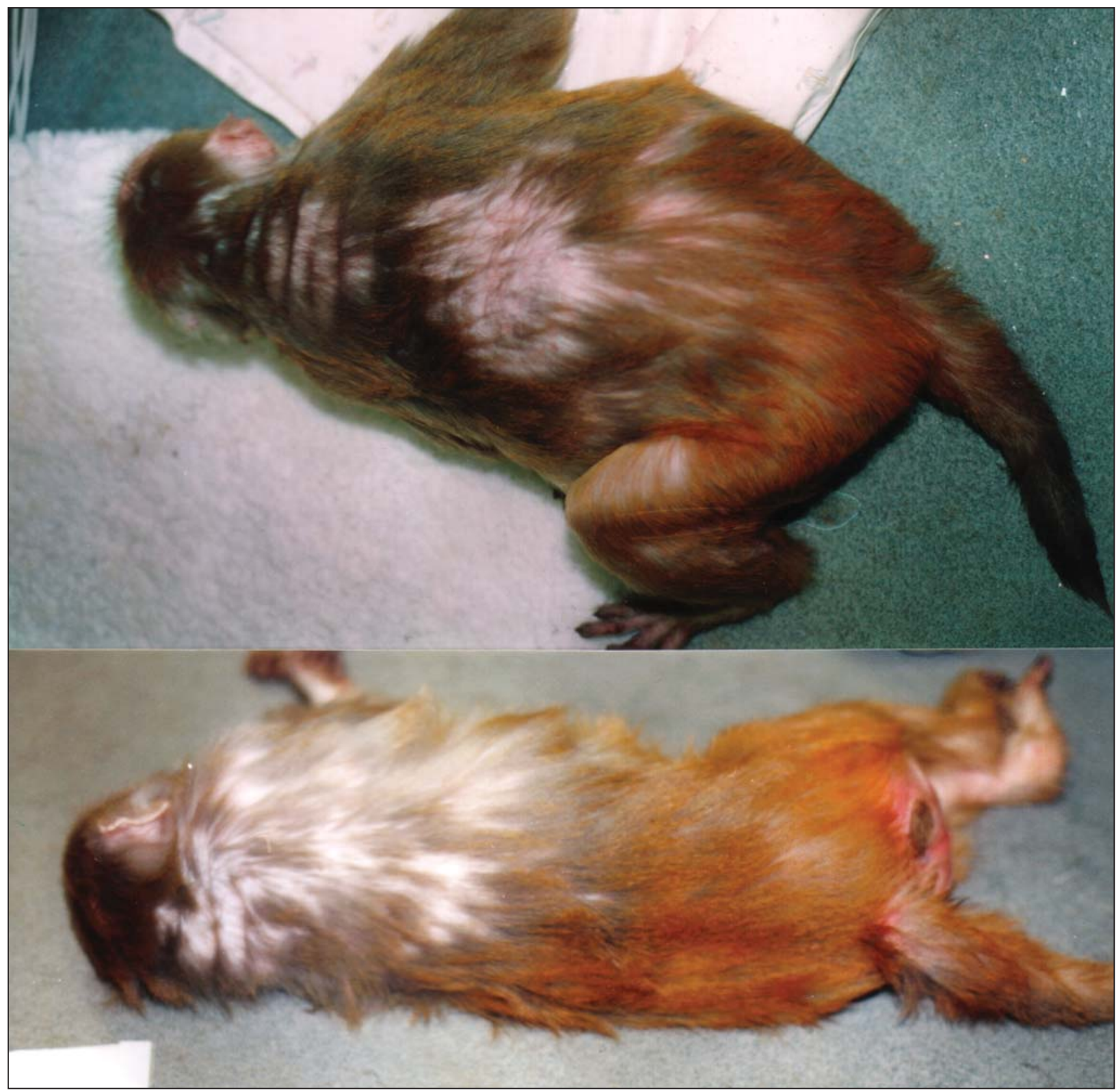

c) Score 3: large patches of alopecia, or numerous small ones that involve a surface between 25 and $50 \%$ of the back

cannibalism (18). Recipients of hair pulling often show fear and/or avoidance reactions, which are likely to reflect poor welfare (13). Quantifying alopecia is thus a potentially useful tool for welfare assessment, especially if used in combination with distress scoring or welfare assessment systems (for examples of these systems, see 19,20).

Alopecia in primates housed under laboratory conditions most commonly results from the pathological intensification of normal grooming behaviour with the plucking of hair, frequently followed by ingestion (for example, in rhesus macaques, Macaca mulatta [13]). In socially housed animals, this is almost exclusively due to allo-grooming (grooming or being groomed by another, in $>97 \%$ of instances) rather than autogrooming or self-grooming (13), and can be very severe, resulting in extensive areas of baldness. In extreme cases where the hair is ingested, this can result in the formation of gastric trichobezoars (hairballs) that may lead to severe clinical illness (21).

Grooming behaviour, from which over-grooming or hair-pulling is derived, has a prominent place in 


\section{Figure 1: continued}

d)

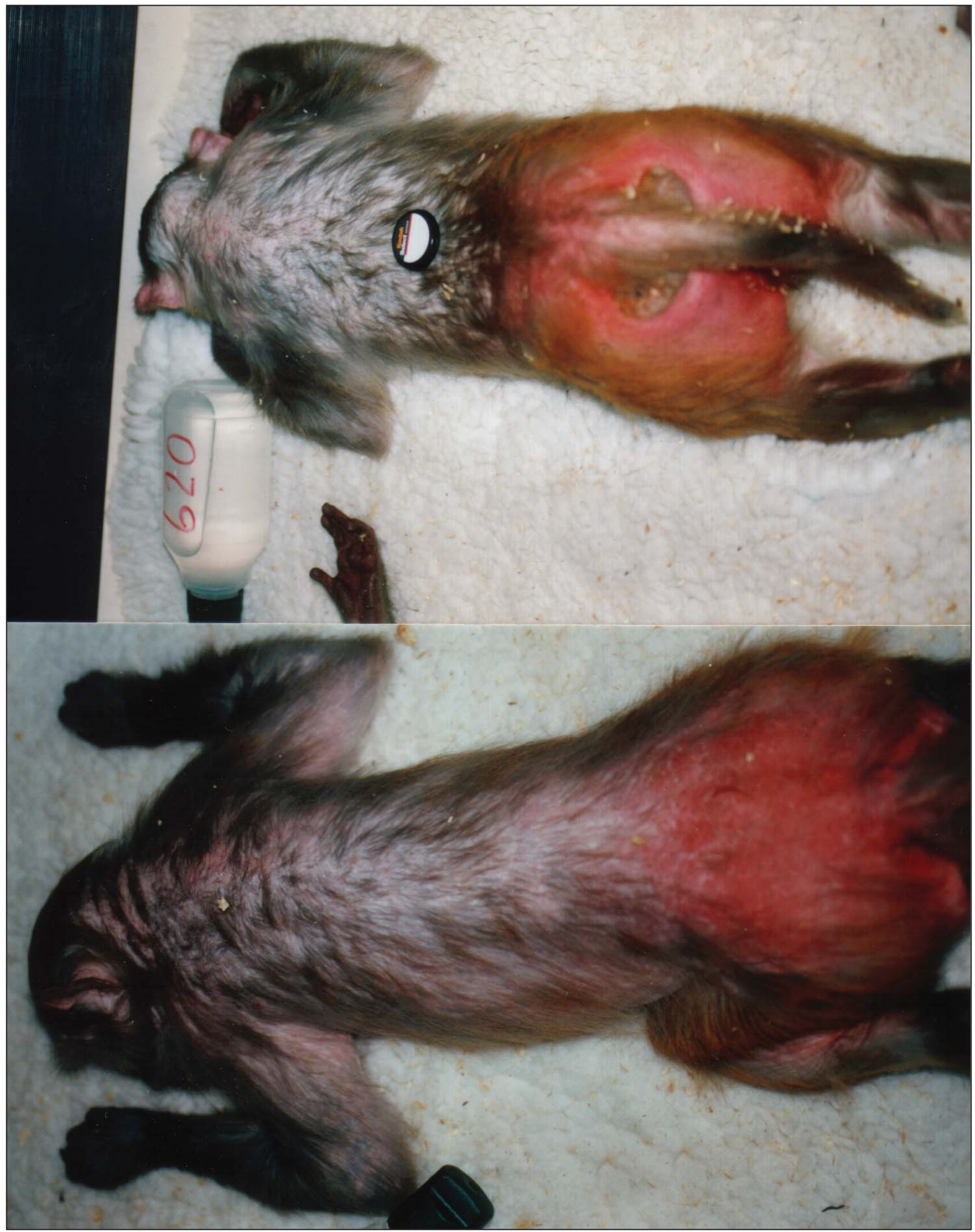

d) Score 4: generalised alopecia (not patchy), more than $50 \%$ of the back 


\section{Figure 1: continued}

e)

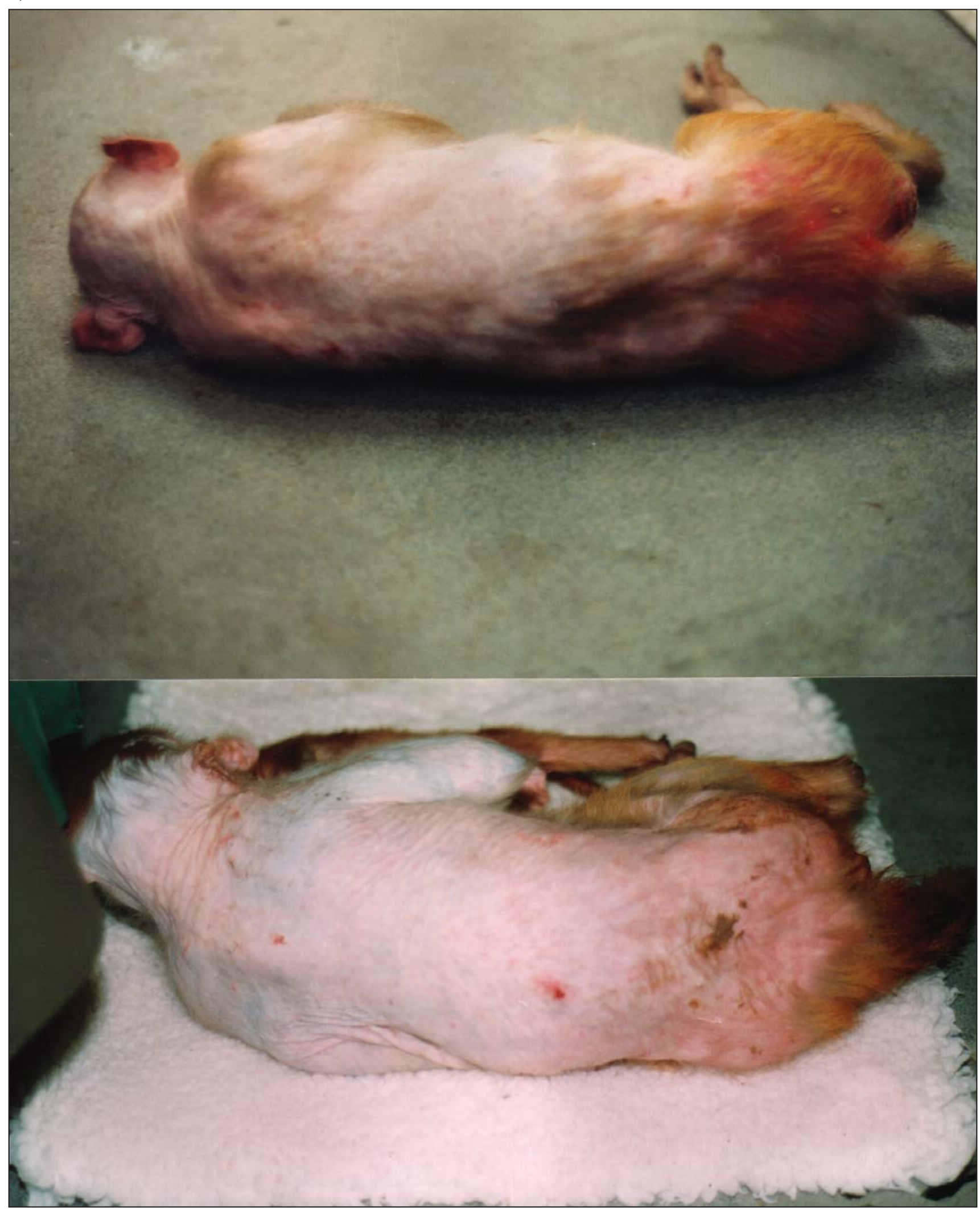

e) Score 5: Back completely bald (more skin visible than hair-coat) 
the normal behavioural repertoire of primates, both in the wild and in captivity (22), so the functions and patterns of allo-grooming in primates have received considerable attention. It is likely that the patterns observed in "normal" grooming behaviour will be reflected in those observed in alopecia due to over-grooming, both in terms of which parts of the body are denuded of hair and which individuals carry out or receive over-grooming. Some researchers claim that allo-grooming is complementary to auto-grooming, focusing on those areas of the body that cannot be reached in auto-grooming (for example 23), so the distribution of bald patches is likely to be a reflection of whether they are a result of allo-grooming or auto-grooming. These researchers have generally ascribed utilitarian functions to allo-grooming, such as parasite and particle removal $(24,25)$, while others point to its social role in the formation and maintenance of social bonds and alliances, as well as in the reconciliation of relationships and reduction of tension following aggressive encounters $(22,26)$. A number of parameters have been known for some time to influence the pattern of allo-grooming observed in primate groups, including an individual's age (27, $28)$ and sex $(22,29)$, its reproductive $(30,31)$ and dominance status $(32,33)$, and the level of aggression in the group $(34,35)$.

Examining the function or cause of hair-pulling and eating with resultant alopecia in captive macaques, Reinhardt et al. (13) point out that it is unlikely to result from nutritional or toxicological factors, as none of their study animals exhibited related clinical symptoms, nor was the pattern of the hair-pulling behaviour uniformly distributed, with younger animals exhibiting more than older ones. They therefore suggest that this behaviour is, as in humans, "a symptom of psychogenic maladjustment". This maladjustment may be brought about by an animal's permanent confinement under stressful conditions of moderate crowding and unchanging environment (13). It may also be derived from the well-documented adverse effects of single-housing on primates, since the most substantial difference that can be made to the life of an individual primate is in the provision of social enrichment in the form of the company of appropriate conspecifics. Monkeys are social animals that live in groups in the wild. Deprivation of social relations will cause stress in a very short time, as social deprivation studies have shown (for further discussion, see 20). The effect is not only seen in the severe consequences demonstrated by the infant deprivation of maternal contact in the classic studies of Harlow et al. (36), but also in the vital buffering effect of the presence of conspecifics when an individual is subjected to a stressful event (37).

Consistent with this, primate hair-pulling is regarded by several authors as being prevalent in captive colonies where there is also other abnormal behaviour and/or stress (13, 38-40), and it has therefore frequently been used as part of a suite of behaviours when assessing psychological well-being (41). The welfare-related nature of this behaviour has been indicated by studies linking changes in levels of hair-pulling with indicators such as the incidence of stereotypic behaviour. For example, Boccia \& Hijazi (42) report a reduction in the frequency of hair-pulling by as much as $88 \%$, accompanied by a reduction of $81 \%$ in stereotypic behaviour, among pig-tailed macaques ( $M$. nemestrina) presented with a foraging task. Similarly, a significant decrease in hair-pulling, together with other self-directed stereotypic behaviours (including self-biting and floating limb), has been reported for long-tailed macaques (M. fascicularis) presented with puzzle feeders (43). The introduction of longlasting environmental modifications, such as training, periodic foraging tasks and social pairing, has produced long-term suppression of hair-pulling, accompanied by sustained reduction in deleterious behaviour, including other stereotypic behaviour and injurious aggression (42-45). The mechanism through which these changes act to suppress negative behaviour is unclear, but they most likely act to increase the animals' control over their environment by presenting opportunities to indulge in novel behaviour or to exhibit existing behaviours in different proportions, thereby contributing to the alleviation of boredom.

The above studies measured the duration or frequency of hair-pulling behaviour, rather than the extent of hair loss resulting from this behaviour. Grooming in rhesus macaques can vary from gentle stroking of the hair, through more intensive contact accompanied by vigorous slapping movements, to the over-grooming or hair-pulling which is the subject of this study. Variation in the intensity of this behaviour may mean that distinguishing between the more intense levels of normal grooming and hair-pulling may prove subjective or difficult if the incidence or duration of this behaviour is being recorded. We believe that the quantification of the resulting hair loss or alopecia may provide a more valuable, practical and objective indicator of welfare.

Therefore, using rhesus macaques, we have developed a scoring system for quantifying this type of alopecia in primates. Scoring or rating systems allow observers to visually classify the appearance of a subject on a purpose-made scale, and have been used in human medicine (46-48) as well as in animal welfare assessments. Scoring systems have been used to quantify specific welfare-relevant variables, such as lameness (49-51), trapping injury (52), chromodacryorrhoea output in rats (53), and the effects of transgenesis on neonatal rodents (54); to formalise judgements about overall pain or stress levels $(55,56)$; and to rank aspects of housing or husbandry $(57,58)$. Good scoring systems are non- 
intrusive, quick and inexpensive, and allow several aspects of state, including the severity and extent of a condition, to be integrated into a single value. However, they also need to be validated before being accepted for widespread use. For instance, each observer must be able to reproduce his or her scores in repeated tests (within-observer reliability; see 50), and different observers must produce scores that both correlate (inter-observer reliability; see 50, 59-61) and show no significant differences in mean value (46). Furthermore, if the scoring system is to be used in a range of different circumstances, or on data collected in different ways, these methodological variations must not affect the scores obtained $(48,51,60)$.

Therefore, our aim was both to develop a scoring system that could readily be used for laboratory primates, and to assess its reliability in terms of observers and observation methods (still photographs versus direct observation of living animals).

\section{Study 1: Development of a Scoring Method}

\section{Methods}

A specific pathogen free (SPF) breeding colony of approximately 200 rhesus macaques (M. mulatta) was used for this study. The animals were housed in single male groups with a varying number (4-11) of adult females and their as yet unweaned offspring (removed from the natal group at between 12 and 18 months). They were kept indoors in typical reinforced stainless steel laboratory cages in the form of three modules (each $1.20 \mathrm{~m}$ wide $\times 1.20 \mathrm{~m}$ deep $\times$ $2.0 \mathrm{~m}$ high) with shelves at two different heights. This allowed the monkeys to access three different levels. The floor was covered with sawdust, which was changed weekly. The cages were washed every 7 to 10 days. The animals were fed three times daily: in the morning, with monkey chow pellets (Harlan Teklad, Indianapolis, IA, USA); around noon, with foraging mixture (peanuts in the shell, flaked maize, dog biscuits, locust beans, sunflower seeds and pine kernels, with boiled eggs in the shell twice weekly and a few dog chocolate drops weekly; [62]), and midafternoon with whole fresh fruit (bananas, apples, oranges, etc.). Water was available ad libitum. The animals were maintained under a 12:12-hour light:dark lighting regime at a regulated temperature $\left(15-24^{\circ} \mathrm{C}\right)$ and humidity $(45 \%-65 \%)$, in accordance with the UK Home Office Code of Practice for the Housing and Care of Animals in Designated Breeding and Supplying Establishments (63).

Photographs were taken of approximately 60 adults, while each subject was sedated with ketamine (Ketaset; Fort Dodge Animal Health, Hedge End, Southampton, UK) for routine annual veterinary health screening. An optical camera and flash were used. During allo-grooming in macaques, dorsal surfaces have been shown to be the single most groomed part of the body (rhesus macaques [M. mulatta], [26]; lion-tailed macaques [M. silenus], and Celebes macaques [M. nigra], [24]), and when pathological levels of over-grooming exist, hair loss is most apparent from these areas. For this reason, and for ease of observation, the dorsum was selected for assessment of hair loss. For photographing, animals were therefore placed supine (to ensure that they kept a free airway) on a piece of Vet Bed (Jørgen KRUUSE A/S, Marslev, Denmark) on a table, with their knees drawn up underneath and their arms in a relaxed position beside the head.

43 of these photographs were then selected, based on their clarity and also to represent the full variability of pelage condition from a complete coat to total baldness. These pictures were used to establish a scoring system that reflected the degree of hair loss. A five-point scale scoring system was developed, based on the coat condition of the animals' backs (Table 1 and Figure 1).

Two independent scorers, not involved in the development of the scoring scale, then applied this scheme to the photographs on separate occasions. One of them was familiar with laboratory rhesus macaques, the other one was naïve and used the set of photographs shown in Figure 1 as reference for scoring the animals. Half-scale points (for example $2.5,3.5$ ) were attributed where a coat condition was judged to fall midway between two scale points.

\section{Table 1: A five-point scoring scale for alopecia in primates}

\section{Score Description}

$1 \quad$ Very good coat condition; complete back cover

2 A few small patches of alopecia $\left(2-5 \mathrm{~cm}^{2}\right)$

3 Large patches of alopecia $\left(\geq 5 \mathrm{~cm}^{2}\right)$, or numerous small ones totalling $25-50 \%$ of the surface of the back

$4 \quad$ Generalised alopecia (not patchy), involving more than $50 \%$ of the back

$5 \quad$ Back completely bald (i.e. more skin visible than hair) 


\section{Statistical analyses}

To check their similarity, the results of the two scorers were compared with a Pearson correlation to check for consistent ordering (inter-observer reliability), and with a paired $t$-test to see if there were differences between the means of their scores. For each test, residuals were checked for normality and homogeneity of variance. The Minitab 13 statistical package (Minitab Inc., State College, PA, USA) was used.

\section{Results}

Inter-observer reliability proved very high $(r=0.92$, $n=43, p<0.001$; see Figure 2), and the scores of the two observers did not differ, i.e. one observer did not score consistently higher than the other $(t=0.33$, $d f=42, p=0.73$ ). The mean scores from the two observers were 2.74 and 2.77 .

\section{Conclusions}

It is clear that the coat condition of laboratory rhesus macaques can reliably be assessed from still photographs. Note that the scores of the two observers were in agreement, although one of them was very familiar with laboratory rhesus monkeys, while the other was not. Hence, it can be concluded that scorers can easily be trained by using the master set of photographs shown in Figure 1.

However, it would often be much more convenient to score primates directly, when awake or restrained, rather than when sedated and/or from photographs. It is not always possible, nor desirable, to sedate animals simply to photograph their dorsum for assessing the degree of their alopecia, so the scoring system would be most useful if it could be extended to non-sedated animals in their home accommodation. To evaluate the application of this system, it was necessary to examine whether a different scale point score would result from an animal being assessed (either from a photograph or direct observation) when sedated and posed, as opposed to moving about in its home accommodation.

\section{Study 2: Comparison of Direct Alopecia Scoring and Scoring from Photographs}

\section{Methods}

This study used the same SPF breeding colony of rhesus macaques, housed in similar single male social groups, as for Study 1. However, since the first study, changes had been made in the housing of the animals (64), which were now accommodated in open rooms containing either cages with the fronts removed or no caging at all. An approximate average total of $22.8 \mathrm{~m}^{3}$ of space was available per group. Each room had an assorted, flexible arrangement of wooden furniture (often diagonally arranged logs/poles), wooden shelves and hanging items (for example, tyres), with a woodchip forage substrate spread on the floor. The walls of the rooms were protected with ceramic tiles or Forex sheeting (Lonza Ltd., Basel, Switzerland), and had protected light and electrical fittings (metal cases). All the rooms had a secondary door. The feeding and watering regime, as well as the lighting, temperature and humidity conditions, were the same as for Study 1. Waste solids were removed from each room every 2 days, and the substrate on the floor of each room changed weekly. The rooms were given a thorough wash down every 10-14 days.

The two observers who took part in this study were different from those involved in Study 1, but, as in Study 1, one was experienced with laboratory rhesus macaques and the other was not. Monkeys from six breeding groups, totalling six males and 29 females, were selected from the colony for this experiment. These were not all the same individuals as in Study 1. The selected groups were the smallest in the colony, and were also part of a separate behavioural study by the two observers, so during direct scoring, they had a high degree of certainty of individual identification. Unlike in Study 1, and to further test the robustness of the scoring system, only whole number scores were attributed; for an animal whose coat condition fell

\section{Figure 2: Study 1: inter-observer correlation in alopecia scores}

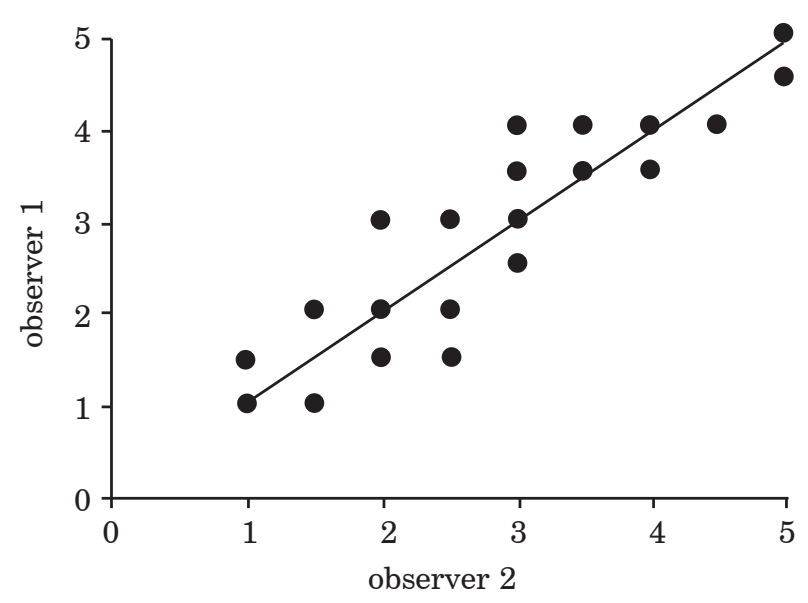

$\mathrm{n}=43 ;$ a number of data points are the same and are therefore superimposed.

$\mathrm{r}_{\mathrm{s}}=0.92 ; \mathrm{p}<0.001$. 
between two scale points, the score given was that which it most closely matched.

\section{Photographic scoring}

All the adult monkeys in the colony were sedated with ketamine, for blood sampling and veterinary examination during the routine annual health screening, as in Study 1. The backs of these monkeys were photographed by using a digital camera with flash for alopecia scoring. They were photographed with a label carrying their name and the date, in the same posture as that described for Study 1. The two observers then independently scored the alopecia at a later time, separately from each other. Results were not discussed until the scoring was complete.

\section{Direct scoring}

The same two observers scored the study animals on another occasion, when the animals were moving about their home enclosures, and with identification assistance from a technician who worked with them daily (and was able to identify all the individuals by tattoos or individual differences). The observers entered the colony separately, to ensure independence from each other, and the animals were scored with reference to the sample photographs (as shown in Figure 1), which the observers carried with them. The accompanying technician was instructed not to coach or prompt the observer on the condition of any animal. As the pattern of hair loss may be asymmet- ric, the score for each animal was not recorded until the whole back, including both left and right flanks, had been seen.

\section{Statistical analyses}

To test for consistency between the two observers, their scores from both photographic and direct observations were tested for correlation and also for significant differences in mean values (as for Study 1). Residuals were checked for normality and homogeneity of variance, as in Study 1, following which non-parametric tests were selected as being the most appropriate. Following this, each observer's photographic and direct observation scores were also compared (Spearman's correlation, $r_{s}$; Wilcoxon signed ranks test for significant differences) to examine within-observer consistency between the two observation methods. All the tests were conducted by using SPSS 11.5 for Windows (SPSS, Inc., Chicago, IL, USA).

\section{Results}

\section{Inter-observer reliability}

As with Study 1 (see above), a very high correlation was found between the scores of the two observers, both when scoring alopecia levels from photographs $\left(r_{s}=0.977, n=35, p<0.001\right.$; see Figure $3 \mathrm{a}$ ), and when using direct observation

Figure 3: Study 2: inter-observer correlations in alopecia scores
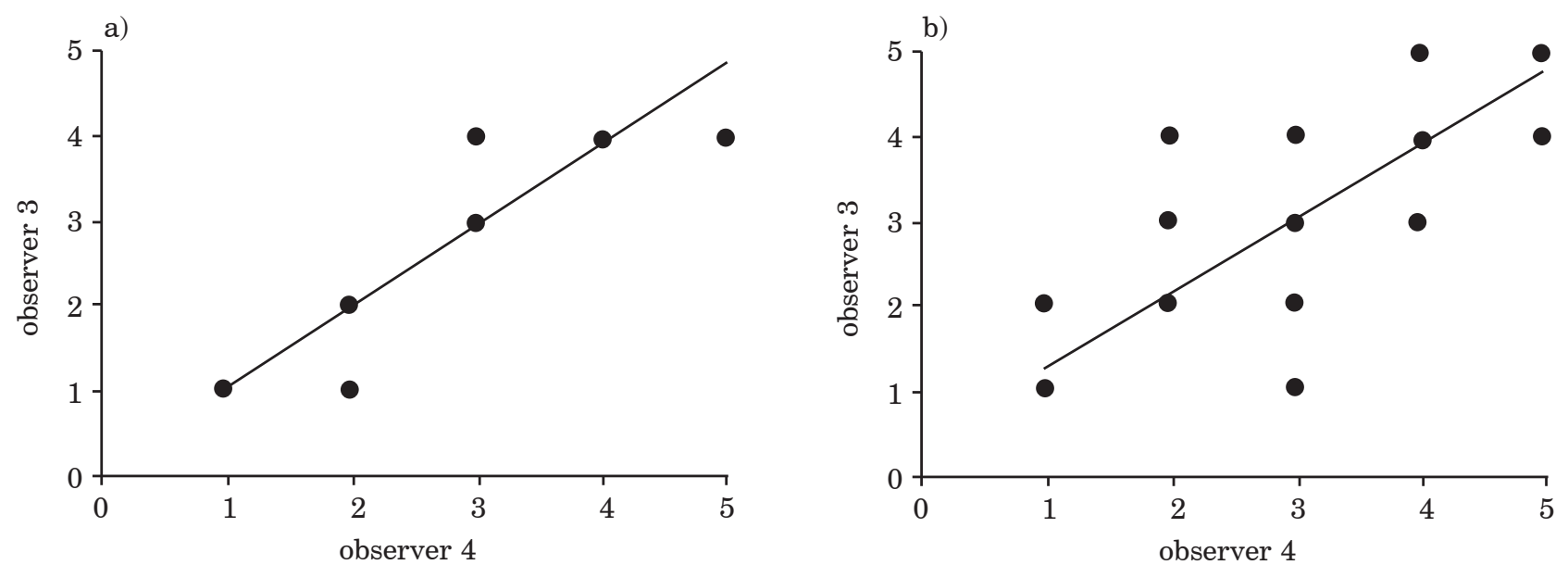

$\mathrm{n}=35 ;$ a number of data points are the same and are therefore superimposed.

a) From photographs; $\mathrm{r}_{\mathrm{s}}=0.98 ; \mathrm{p}<0.001$.

b) From direct observation; $\mathrm{r}_{\mathrm{s}}=0.87$; $\mathrm{p}<0.001$. 
$\left(r_{s}=0.869, n=35, p<0.001\right.$; see Figure 3b). Wilcoxon signed ranks tests also revealed no significant difference between the scoring of both observers from photographs $(Z=-0.577, p=0.564$, number of pairs $=35$, mean scores $=2.34$ and 2.31 ) and direct observation $(Z=-0.924, p=0.356$, number of pairs $=35$, mean scores $=2.23$ and 2.34).

\section{Consistency between observation methods}

Further Spearman's correlations and Wilcoxon signed ranks tests showed significant correlations, and no significant differences, between the scoring by each observer from photographs and direct observation (Observer 3: $r_{s}=0.856, n=35, p<0.001$, see Figure 4a; Wilcoxon's $Z=-0.943, p=0.346$, number of pairs $=35$. Observer 4: $r_{s}=0.909, n=35$, $p<0.001$, see Figure 4b; Wilcoxon's $Z=-0.302$, $p=0.763$, number of pairs $=35$ ).

\section{Conclusions}

The high correlations and lack of significant differences between all the measures in Study 2 demonstrated the reliable nature of the scoring system developed in Study 1. As such, interobserver and inter-method reliability were demonstrated, both for scoring from photographs of posed, sedated animals, and from direct observation of non-sedated animals in their home cage, and for whole-number scoring. It might be expected that there would be small differences between scores from photographs and direct observation, not least due to the different body postures and lighting conditions. Under flash photography, the light-coloured under-fur of rhesus macaques can appear like skin, and in some cases may result in the slight exaggeration of hair-loss compared with the less intense and less direct lighting in the animals' home cages. It was reassuring that no significant effects were found.

\section{General Discussion}

This study demonstrates that the proposed scoring system provides a practical method for assessing alopecia and hair loss in primates that are either sedated or free-moving. While the presence and degree of alopecia remains to be correlated against quantitative assessments of stress from physiological and/or behavioural measures (the subject of continuing research by the authors), the repeated, sequential use of this scoring system will enable researchers and animal care staff to carry out simple quantified assessments of the animal welfare benefits of changes in their environment and husbandry, especially if used in combination with other welfare assessment systems.

In addition, provided that inter-observer consistency was demonstrated (as in this study), this scoring system could be applied by more than one assessor in the same study, and could also allow results to be confidently compared from one study to the next (c.f., for example, reference 50). It benefits from being both an efficient use of staff time (the

\section{Figure 4: Study 2: correlations in alopecia scores from photographic and direct methods}
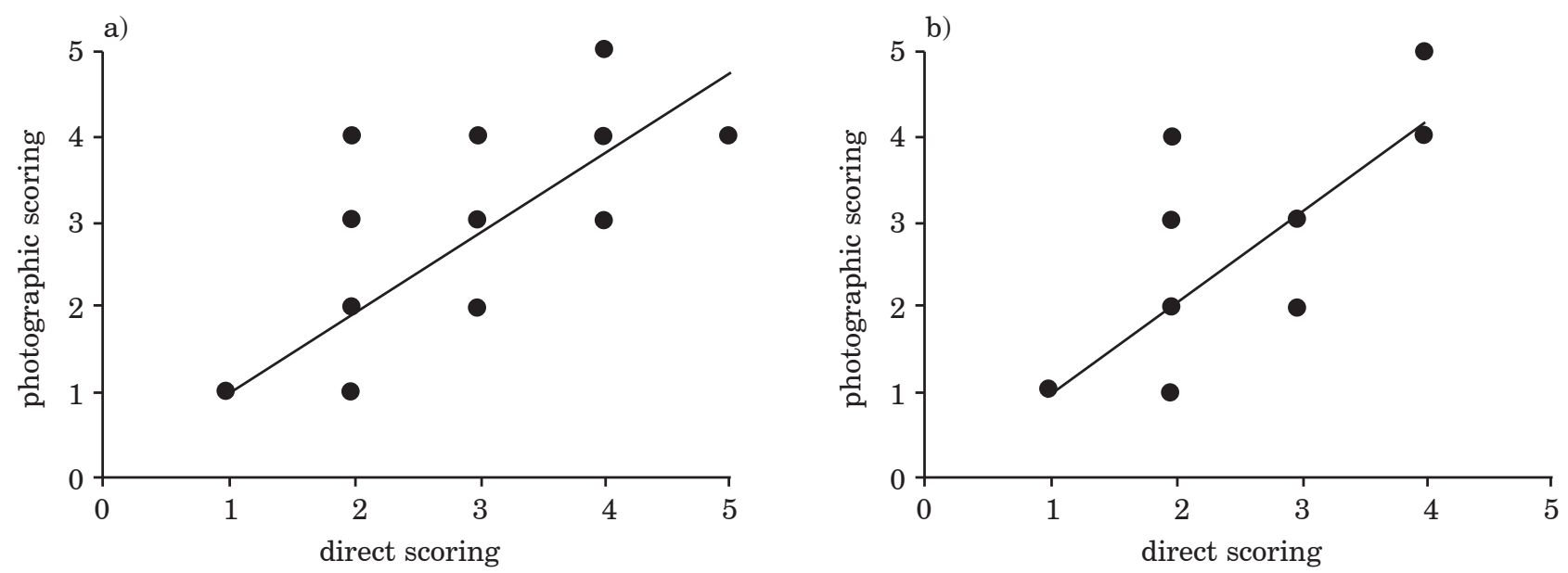

$\mathrm{n}=35$; a number of data points are the same and are therefore superimposed.

a) Observer 3; $\mathrm{r}_{\mathrm{s}}=0.86, \mathrm{p}<0.001$.

b) Observer 4; $\mathrm{r}_{\mathrm{s}}=0.91, \mathrm{p}<0.001$. 
coat scoring system being less time consuming than observing and recording over-grooming behaviour) and easy to implement (since animals are carefully observed and then given the closest alopecia score based on the reference photographs). For example, in Study 2, only approximately 30 minutes was necessary to train an observer. The training involved:

1. distinguishing between genuine baldness and what appeared to be bare patches as a result of erect or ruffled hair;

2. familiarisation with the reference photographs;

3. identification of individual monkeys;

4. ensuring the whole dorsum (including both flanks) was assessed; and

5. accurate completion of data sheets.

The observation of hair-pulling behaviours would be required to identify which animals performed such behaviour and which events triggered it. This type of information is necessary to determine the ultimate and proximate causes of the behaviour and thus for designing strategies to reduce it and improve welfare. Observation of grooming behaviour would also yield information about positive and negative relationships between individual animals, and would increase the probability of noticing other behavioural problems, injuries, etc., which might be indicative of poor welfare.

In the future, it is intended that this scoring system could also form the basis of similar scoring systems for use on the pelages of other animals - such as barbered laboratory rats and mice - where typically, as with primates, duration and frequency of hair-pulling behaviour is measured (4). Hair loss scoring has been used in rodents to examine barberspecific patterns and the extent of hair loss by using custom computer software (5). The proposed system avoids the need for such specialised tools, while permitting the examination of gross changes in hair loss in primates.

\section{Acknowledgements}

The authors would like to thank the two anonymous referees for their helpful and incisive comments on this paper, and the management and animal care staff at Harlan Hillcrest, as well as those that assisted as observers in this study (Carolina Marin and Daniela Bonacic), Julian Howe for additional help with data collection, and Manuel Berdoy for advice on analysis. Also our funders: UFAW, LASA, and Oxford University Research Development Fund.
Received 19.10.04; received in final form 10.12.04; accepted for publication 17.12.04.

\section{References}

1. van Hoek, C.S. \& King, C.E. (1997). Causation and influence of environmental enrichment on feather picking of the crimson-bellied conure (Pyrrhura perlata perlata). Zoo Biology 16, 161-172.

2. Oden, K., Vestergard, K.S. \& Algers, B. (1999). Agonistic behaviour and feather plucking in singlesexed and mixed groups of laying hens. Applied Animal Behaviour Science 62, 219-231.

3. Mason, G.J. (1994). Tail-biting in mink (Mustela vison) is influenced by age at removal from the mother. Animal Welfare 3, 305-311.

4. Sarna, J.R., Dyck, R.H. \& Whishaw, I.Q. (2000). The Dalila effect: C57BL6 mice barber whiskers by plucking. Behavioural Brain Research 108, 39-45.

5. Garner, J.P., Weisker, S.M., Dufour, B. \& Mench, J.A. (2004). Barbering (fur and whisker trimming) by laboratory mice as a model of human trichotillomania and obsessive-compulsive spectrum disorders. Comparative Medicine 54, 216-224.

6. Venatesan, R., Nagarajan, P., Mahesh Kumar, M.J. \& Majumdar, S.S. (2004). Environment- and seasoninfluenced alopecia in juvenile rhesus macaques. Laboratory Primate Newsletter 43, 1-2.

7. Messenger, A.G. (2003). The control of hair growth. In Disorders of Hair Growth: Diagnosis and Treatment (ed. E.A. Olsen), pp. 39-58. New York, NY, USA: McGraw Hill.

8. Meehan, C.L., Garner, J.P. \& Mench, J.A. (2003). Isosexual pair housing improves the welfare of young Amazon parrots. Applied Animal Behaviour Science, New York 81, 73-88.

9. Morishita, T.Y., Aye, P.P. \& Harr, B.S. (1999). Crop impaction resulting from feather ball formation in caged layers. Avian Diseases 43, 160-163.

10. Shively, C.A., Grant, K.A. \& Register, T.C. (2002). Effects of long-term moderate alcohol consumption on agonistic and affiliative behavior of socially housed female cynomolgus monkeys (Macaca fascicularis). Psychopharmacology 165, 1-8.

11. Boccia, M.L. (1989). Preliminary report on the use of a natural foraging task to reduce aggression and stereotypies in socially housed pigtail macaques. Laboratory Primate Newsletter 28, 3-4.

12. Tay, Y.K., Levy, M.L. \& Metry, D.W. (2004). Trichotillomania in childhood: case series and review. Pediatrics 113, E494-E498.

13. Reinhardt, V., Reinhardt, A. \& Houser, D. (1986). Hair pulling and eating in captive rhesus monkey troops. Folia Primatologica 47, 158-164.

14. Joubert, A.F. \& Stein, D.J. (1999). Citalopram and anxiety disorders. Reviews in Contemporary Pharmacotherapy 10, 79-131.

15. Gupta, M.A. \& Gupta, A.K. (2001). The use of antidepressant drugs in dermatology. Journal of the European Academy of Dermatology and Venereology 15, 512-518.

16. Figgitt, D.P. \& McClellan, K.J. (2000). Fluvoxamine: an updated review of its use in the management of adults with anxiety disorders. Drugs 60, 925-954.

17. Christenson, G.A. \& Mansueto, C.S. (1999). Trichotillomania: descriptive characteristics and phenomenology. In Trichotillomania (ed. D.J. Stein, G.A. Christenson \& E. Hollander), pp. 1-41. Washington, 
DC, USA: American Psychiatric Press, Inc.

18. Kjaer, J.B. \& Vestergaard, K.S. (1999). Development of feather pecking in relation to light intensity. Applied Animal Behaviour Science 62, 243-254.

19. Wolfensohn, S.E. \& Lloyd, M. (2003). Handbook of Laboratory Animal Management and Welfare, 3rd edn, 416pp. Oxford, UK: Blackwell Publishing Ltd.

20. Wolfensohn, S.E. \& Honess, P.E. (2005). Handbook of Primate Husbandry and Welfare, 168pp. Oxford, UK: Blackwell Publications.

21. Mook, D.M. (2002). Gastric trichobezoars in a rhesus macaque (Macaca mulatta). Comparative Medicine 52, 560-562.

22. Rosenblum, L.A., Kaufman, I.C. \& Stynes, A.J. (1966). Some characteristics of adult social and autogrooming patterns in two species of macaque. Folia Primatologica 4, 438-451.

23. Barton, R. (1985). Grooming site preferences in primates and their functional implications. International Journal of Primatology 6, 519-532.

24. Hutchins, M. \& Barash, D.P. (1976). Grooming in primates: implications for its utilitarian function. Primates 17, 145-150.

25. Freeland, W.J. (1981). Functional aspects of primate grooming. Ohio Journal of Science 81, 173-177.

26. Boccia, M.L. (1983). A functional analysis of social grooming patterns through direct comparison with self-grooming in rhesus monkeys. International Journal of Primatology 4, 399-418.

27. Goy, R.W., Kraemer, G. \& Goldfoot, D. (1988). Biological influences on grooming in nonhuman primates. Annals of the New York Academy of Sciences 525, 56-58.

28. Drickamer, L.C. (1976). Quantitative observations of grooming behaviour in free-ranging Macaca mulatta. Primates 17, 323-335.

29. Mitchell, G. \& Tokunaga, D.H. (1976). Sex differences in nonhuman primate grooming. Behavioural Processes 1, 335-345.

30. Rowell, T.E. (1968). Grooming by adult baboons in relation to reproductive cycles. Animal Behaviour 16, 585-588.

31. Domb, L.G. \& Pagel, M. (2001). Sexual swellings advertise female quality in wild baboons. Nature, London 410, 204-206.

32. Seyfarth, R.M. (1980). The distribution of grooming and related behaviours among adult female vervet monkeys. Animal Behaviour 28, 798-813.

33. Schino, G. (2001). Grooming, competition and social rank among female primates: a meta-analysis. Animal Behaviour 62, 265-271.

34. Terry, R.L. (1970). Primate grooming as a tension reduction mechanism. The Journal of Psychology 76, 129-136.

35. Barrett, L., Gaynor, D. \& Henzi, S.P. (2002). A dynamic interaction between aggression and grooming reciprocity among female chacma baboons. Animal Behaviour 63, 1047-1053.

36. Harlow, H.F., Harlow, M.K. \& Suomi, S.J. (1971). From thought to therapy: lessons from a primate laboratory. American Scientist 59, 538-549.

37. Vogt, J.L., Coe, C.L. \& Levine, S. (1981). Behavioural and adrenocorticoid responsiveness of squirrel monkeys to a live snake: is flight necessarily stressful? Behavioural and Neural Biology 32, 391-405.

38. Roberts, J.A. (1992). Behavioral observation in a macaque breeding colony: assessment of enrichment strategies. In XIVth Congress of the International Primatological Society, Abstracts, pp. 167-168.
39. Nash, L.T., Fritz, J., Alford, P.A. \& Brent, L. (1999). Variables influencing the origins of diverse abnormal behaviors in a large sample of captive chimpanzees (Pan troglodytes). American Journal of Primatology 48, 15-29.

40. Goodwin, J. (1997). The application, use, and effects of training and enrichment variables with Japanese snow macaques (Macaca fuscata) at the Central Park Wildlife Center. Regional Conference Proceedings, pp. 510-515. Silver Spring, MD, USA: American Zoo and Aquarium Association.

41. Lutz, C., Well, A. \& Novak, M. (2003). Stereotypic and self-injurious behaviour in rhesus macaques: a survey and retrospective analysis of environment and early experience. American Journal of Primatology 60, 1-15.

42. Boccia, M.L. \& Hijazi, A.S. (1998). A foraging task reduces agonistic and stereotypic behaviours in pigtail macaque social groups. Laboratory Primate Newsletter 37, 1-5.

43. Watson, L.M. (1992). Effect of an enrichment device on stereotypic and self-aggressive behaviours in singly-caged macaques: a pilot study. Laboratory Primate Newsletter 31, 8-10.

44. Statz, L.M. \& Borde, M. (2001). Pairing successes with male cynomolgus macaques after vasectomy. AALAS Official Program, 102-103.

45. Boccia, M.L. (1989). Long-term effects of a natural foraging task on aggression and stereotypies in socially housed pigtail macaques. Laboratory Primate Newsletter 28, 18-9.

46. Fitzgerald, D.A., VanAsperen, P.P., Lam, A.H., de Silva, M. \& HendersonSmart, D.J. (1996). Chest radiograph abnormalities in very low birthweight survivors of chronic neonatal lung disease. Journal of Paediatrics and Child Health 32, 491-494.

47. Donner-Banzhoff, N., Beck, C., Meyer, F., Werner, J.A. \& Baum, E. (2002). Clinical findings in patients presenting with sore throat: a study on interobserver reliability. Family Practice 19, 466-468.

48. Bird, P., Ejbjerg, B., McQueen, F., Ostergaard, M., Lassere, M. \& Edmonds, J. (2003). OMERACT rheumatoid arthritis magnetic resonance imaging studies. Exercise 5: An international multicenter relability study using computerized MRI erosion volume measurements. Journal of Rheumatology 30, $1380-1384$

49. Kestin, S.C., Gordon, S., Su, G. \& Sorensen, P. (2001). Relationships in broiler chickens between lameness, liveweight, growth rate and age. Veterinary Record 148, 195-197.

50. Garner, J.P., Falcone, C., Wakenell, P., Martin, M. \& Mench, J.A. (2002). Reliability and validity of a modified gait scoring system and its use in assessing tibial dyschondroplasia in broilers. British Poultry Science 43, 355-363.

51. Chaplin, S. \& Munksgaard, L. (2001). Evaluation of a simple method for assessment of rising behaviour in tethered dairy cows. Animal Science 72, 191-197.

52. Hubert, G.F., Wollenberg, G.K., Hungerford, L.L. \& Bluett, R.D. (1999). Evaluation of injuries to Virginia opossums captured in the EGG (TM) trap. Wildlife Society Bulletin 27, 301-305.

53. Mason, G.J., Wilson, D., Hampton, C. \& Würbel, H. (2004). Non-invasively assessing disturbance and stress in laboratory rats by scoring chromodacryorrhoea. ATLA 32, 153-159.

54. Lloyd, M., Wolfensohn, S. \& Thornton, P. (2000). Quantitative assessment of welfare in experimental 
animals: the development and use of scoring systems. In Progress in the Reduction, Refinement and Replacement of Animal Experimentation (ed. M. Balls, A-M. van Zeller \& M.E. Halder), pp. 1107-1117. Amsterdam, The Netherlands: Elsevier.

55. Ottway, D.S. \& Hawkins, D.M. (2003). Cat housing in rescue shelters: a welfare comparison between communal and discrete-unit housing. Animal Welfare 12, 173-189.

56. Flecknell, P.A. \& Roughan, J.V. (2004). Assessing pain in animals - putting research into practice. Animal Welfare 13, S71-S75.

57. Bartussek, H. (2001). An historical account of the development of the animal needs index ANI-35L as part of the attempt to promote and regulate farm animal welfare in Austria: an example of the interaction between animal welfare science and society. Acta Agriculturae Scandinavica Section A - Animal Science, 34-41.

58. Bartussek, H. (1999). A review of the animal needs index (ANI) for the assessment of animals' wellbeing in the housing systems for Austrian proprietary products and legislation. Livestock Production Science 61, 179-192.
59. Martin, P. \& Bateson, P. (1993). Measuring Behaviour: An Introductory Guide, 2nd edn, 222pp. Cambridge, UK: Cambridge University Press.

60. Macnab, A.J., Levine, M., Glick, N., Phillips, N., Susak, L. \& Elliott, M. (1994). The Vancouver Sedative Recovery Scale for children — validation and reliability of scoring based on videotaped instruction. Canadian Journal of Anaesthesia 41, 913-918.

61. Ayral, X., Gueguen, A., Ike, R.W., Bonvarlet, J.P., Frizziero, L., Kalunian, K., Moreland, L.W., Myers, S., O'Rourke, K.S., Roos, H., Altman, R. \& Dougados, M. (1998). Inter-observer reliability of the arthroscopic quantification of chondropathy of the knee. Osteoarthritis and Cartilage 6, 160-166.

62. Davys, J.S. (1995). The development of a forage diet for a breeding colony of rhesus monkeys. Animal Technology 46, 63-66.

63. Home Office (1995). Code of Practice for the Housing and Care of Animals in Designated Breeding and Supplying Establishments, 56pp. London, UK: HMSO.

64. Wolfensohn, S.E. (2004). Social housing of large primates: methodology for refinement of husbandry and management. ATLA 32, 149-151. 